\title{
Isolation of phosphate solubilizing fungi from rhizosphere soil and its effect on seed growth parameters of different crop plants
}

\author{
Murali Mahadevamurthy, Thriveni M Channappa, Manjula Sidappa, Mythrashree S Raghupathi, Amruthesh K Nagaraj ${ }^{*}$ \\ Applied Plant pathology Laboratory, Department of Studies in Botany, University of Mysore, Manasagangotri, Mysore- 570 006, India.
}

\section{ARTICLE INFO \\ Article history: \\ Received on: 14/07/2016 \\ Revised on: 04/08/2016 \\ Accepted on: 26/08/2016 \\ Available online: 05/11/2016}

Key words:

Phosphate solubilization,

Penicillium sp., seed growth

parameters, crop plants.

\begin{abstract}
A total of twenty two rhizospheric fungi were isolated from different rhizosphere soil of healthy crop plants of Mysore, Karnataka, India and tested for their efficacy to solubilize phosphates in PKV medium. Among the isolates, Penicillium sp. RFUOM 14 solubilized phosphates by producing clear halo zone around the colony. Further, Penicillium sp. RFUOM 14 inducers were treated for different crop plants and observed that the treatments enhanced seed germination and seedling vigor. Among the treatments, conidial suspension treated for $6 \mathrm{~h}$ showed maximum of $85.75 \%, 80 \%$ and $83 \%$ of seed germination and seedling vigor $985.25,523$ and 673.5 in pearl millet, brinjal and tomato, respectively.
\end{abstract}

\section{INTRODUCTION}

Phosphorous $(\mathrm{P})$ is an essential macronutrient for plant growth. It is one of the major limiting factors for crop production on many tropical and sub tropical soils [1]. It plays an important role in energy transfer reactions, respiration and photosynthesis. In plants, phosphorous increases the strength of cereal straw, promotes flower formation and fruit production, stimulates root development and also essential for seed formation [2]. It also plays a role in root development, stalk and stem strength, flower and seed formation, maturity and production, crop quality and resistance to plant diseases. There are evidences of occurrence of rhizospheric phosphorous solubilizing microorganisms which play a key role in soil phosphorous dynamics and subsequent availability of phosphorous to plants [3].

Although large amounts of soluble phosphates are applied to soil as fertilizers, plants are able to use only a small portion of the applied phosphate and the remains are rapidly immobilized and becomes unavailable to plants. Many soil fungi and bacteria are known to solubilize inorganic phosphate and are termed as Phosphate Solubilizing Microorganisms (PSMs). These PSMs

* Corresponding Author

Phone No: +910821 2419760; Fax No: +910821 2419759

Email:dr.knamruthesh@ botany.uni-mysore.ac.in play an important role in supplementing phosphorous to the plants, allowing a sustainable use of phosphate fertilizers [4]. Since, plants can absorb phosphate only in soluble form; the transformation of insoluble phosphate into soluble form is carried out only by microbes present in the soil. Several groups of microorganisms including fungi, bacteria and actinomycetes are known as efficient fixed P solubilizers [5].

Fungi are the important components of soil microbes typically constituting more of the soil biomass than bacteria, depending on soil depth and nutrient conditions. Fungi have been reported to have greater ability to solubilize insoluble phosphate than bacteria [6]. A wide range of soil fungi are reported to solubilize insoluble phosphorous such as Aspergillus niger and Penicillium sp., which are the most common fungi capable of phosphate solubilization [7]. For a sustainable agricultural system, microbial solubilization of rock phosphate and its use in agriculture is receiving greater attention. Many soil fungi and bacteria can solubilize inorganic phosphate into soluble forms through the process of acidification, chelation, exchange reactions and production of organic acids [8]. Application of PSMs in the field has been reported to increase crop yield. Chemical fertilizers pose health hazard and affect the microbial population in soil by degrading the physical structure of the soil leading to lack of oxygen in the plant root zone besides being quite expensive and making the cost of production high. 
Whereas naturally, the majority of the microorganisms distributed around plant root surface have a role in the decomposition of organic matter and some way suppress deleterious microorganisms distributed around plant root surface and have role on uptake of nutrients and decomposition of organic matter [9]. Hence, the main objective of the present study was to isolate phosphate solubilizing fungi from rhizosphere and to test their ability to increase seed germination and seedling vigor of pearl millet, tomato and brinjal plants.

\section{MATERIALS AND METHODS}

\subsection{Collection of soil sample}

The rhizospheric soil samples were collected from different regions of Mysore district of Karnataka from various crop plants. The collected soil samples were brought into laboratory, dried and used for further studies.

\subsection{Isolation and identification of rhizosphere fungi}

Each of the rhizosphere soil samples were subjected for serial dilution on potato dextrose agar (PDA) medium and incubated for 7 days at $25{ }^{\circ} \mathrm{C}$. After 7 days of incubation, each individual fungal colony was picked from the edge with a sterile fine tipped inoculation needle and transferred on to the PDA medium [10]. The fungi were identified based on the morphological, conidial (fruiting bodies) and culture characters. The classifications of the fungi were carried out following standard procedures. All the isolated fungi were named and maintained in test tubes and Petri plates containing PDA media and used for further studies.

\subsection{Screening of phosphate solubilizing fungi}

In order to detect phosphate-solubilizing fungi, each of the isolated rhizosphere fungi were inoculated on Pikovskaya's agar (PVK, containing per litre: $0.5 \mathrm{~g}$ yeast extract, $10 \mathrm{~g}$ dextrose, $5 \mathrm{~g} \mathrm{Ca}_{3}\left(\mathrm{PO}_{4}\right)_{2}, 0.5 \mathrm{~g}\left(\mathrm{NH}_{4}\right)_{2} \mathrm{SO}_{4}, 0.2 \mathrm{~g} \mathrm{KCl}, 0.1 \mathrm{~g} \mathrm{MgSO}_{4} .7 \mathrm{H}_{2} \mathrm{O}$, $0.0001 \mathrm{~g} \mathrm{MnSO}_{4} \cdot \mathrm{H}_{2} \mathrm{O}, 0.0001 \mathrm{~g} \mathrm{FeSO}_{4} \cdot 7 \mathrm{H}_{2} \mathrm{O}$ and $15 \mathrm{~g}$ agar, $\mathrm{pH}$ 7.2). The plates were incubated at $25 \pm 2^{\circ} \mathrm{C}$ for 10 days [11]. Fungi capable of producing a halo/ clear zone due to solubilization were selected as potential phosphate solubilizers and used for further studies.

\subsection{Preparation of inducers from Penicillium sp. RFUOM 14}

\subsubsection{Conidial suspension (CS)}

Phosphate solubilizing fungus Penicillium sp. RFUOM 14 was mass multiplied on PDA medium and incubated at $25 \pm 2{ }^{\circ} \mathrm{C}$ for 7 days. After incubation, an aliquot of $10 \mathrm{ml}$ of sterile distilled water (SDW) was added to each of the culture plates and gently shaken to dislodge conidia from the culture surface and collected in $250 \mathrm{ml}$ conical flask. The collected conidial suspension were further passed through a few layers of cheese cloth and centrifuged. The resulting pellate was re-suspended in SDW and the concentration of conidia was adjusted to $1 \times 10^{8} \mathrm{cfu} / \mathrm{ml}$ using Haemocytometer [12].

\subsubsection{Culture Filtrate $(\mathrm{CF})$}

Mycelial discs (10-15 numbers; $5 \mathrm{~mm}$ diameter each) of Phosphate solubilizing fungus Penicillium sp. RF UOM 14 grown on PDA medium obtained from the actively growing margins were transferred to $500 \mathrm{ml}$ conical flasks containing $200 \mathrm{ml}$ of potato dextrose broth (PDB) (pH 6.5). The conical flasks inoculated with Penicillium sp. RFUOM 14 was incubated at $25 \pm 2{ }^{\circ} \mathrm{C}$ for $10-12$ days. The resulting culture filtrate was filtered through few layers of cheesecloth and the filtrate was centrifuged at $8000 \mathrm{rpm}$ for $15 \mathrm{~min}$. The supernatant was collected and sterilized by filtration through $0.22 \mu \mathrm{m}$ Millipore membrane. The sterile filtrates were collected and stored at $4{ }^{\circ} \mathrm{C}[13]$.

\subsection{Inducer treatment}

The seeds of pearl millet, brinjal and tomato were surface-sterilized with sodium hypochlorite for $2 \mathrm{~min}$, and rinsed thoroughly in sterile distilled water (SDW) 2-3 times. The sterilized seeds were treated with conidial suspension and culture filtrate of phosphate solubilizing fungi Penicillium sp. RF UOM 14 at $1 \times 10^{8} \mathrm{cfu} / \mathrm{ml}$ and culture filtrate by mixing 400 seeds of each of the samples with $50 \mathrm{ml}$ conidial suspension. Treated seeds were kept at $25 \pm 2^{\circ} \mathrm{C}$ in a rotary shaker for $3 \mathrm{~h}$ and $6 \mathrm{~h}$ separately to facilitate the penetration of the inducer. After incubation, the seeds were air dried aseptically under laboratory conditions $\left(25 \pm 2^{\circ}\right.$ C) and used for further studies. Seeds treated with SDW served as untreated control.

\subsection{Effect of seed treatment with inducers of Penicillium sp. RFUOM 14 on seed germination and seedling vigor}

The inducer treated and untreated seeds (four replicates of 100 seeds each) were plated equidistantly on three layers of moistened blotting paper discs in Petri dishes to evaluate percentage germination [14] and another set of treated and untreated seeds were subjected to between paper method to record seedling vigor [15]. The experiment consisted of four replications of 100 seeds (50 seeds in eight towels) and was repeated thrice. After 7 days (pearl millet) and 14 days (for brinjal and tomato), percentage germination and vigor were calculated using the formula:

Vigor index $=$ Seed germination $(\%) \times[$ Mean Root Length + Mean Shoot Length]

\subsection{Statistical analysis}

Data from four replicates were analyzed for each experiment and subjected to arcsine transformation and analysis of variance (ANOVA) using SPSS Inc. 16.0. Significant effects of treatments were determined by $\mathrm{F}$ values $(\mathrm{P}<0.05)$. Treatment means were separated by Tukey's honestly significant differences (HSD) test. 


\section{RESULTS}

\subsection{Isolation and identification of rhizosphere fungi}

A total of 22 rhizospheric fungi were isolated from the rhizosphere soil of different healthy crop plants of Mysore district of Karnataka, India (Table 1). The isolated fungi belonged to the genera of Aspergillus, Fusarium, Nigrospora, Penicillium, Pestalotiopsis and Trichoderma. All the isolated rhizosphere fungi were serially numbered (RFUOM 01 to RFUOM 22) and maintained on Petri plates and test tubes containing PDA medium.

Table 1: Fungi isolated from the rhizosphere soil of different crop plants

\begin{tabular}{lccccc}
\hline \multirow{2}{*}{$\begin{array}{c}\text { Rhizosphere } \\
\text { fungi }\end{array}$} & \multicolumn{4}{c}{ Place of collection } & No. of \\
\cline { 2 - 5 } & $\begin{array}{c}\text { T. } \\
\text { Narsipur }\end{array}$ & $\begin{array}{c}\text { H.D. } \\
\text { Kote }\end{array}$ & $\begin{array}{c}\text { K.R. } \\
\text { Nagar }\end{array}$ & Hunsur & \\
\hline Aspergillus sp. & 0 & 2 & 1 & 1 & 04 \\
Fusarium sp. & 1 & 1 & 1 & 2 & 05 \\
Nigrospora sp. & 1 & - & 2 & - & 03 \\
Penicillium sp. & 2 & 1 & 1 & 1 & 04 \\
Pestalotiopsis sp. & - & 1 & - & - & 02 \\
Trichoderma sp. & 2 & 1 & - & 1 & 04 \\
Total & 06 & 06 & 05 & 05 & 22 \\
\hline
\end{tabular}

Figures inside the column represent the total number of rhizosphere fungal isolates

\subsection{Screening of phosphate solubilizing fungi}

All the isolated rhizosphere fungi were sub cultured on Pikovskaya's agar medium to test their ability to solubilize the organic phosphate in the medium. Among the tested fungal isolates, Penicillium sp. RFUOM 14 was capable of producing a halo/ clear zone due to solubilization of phosphate (Fig. 1), while all the other fungal isolates were unable to produce clear/ halo zone in the PKV medium.

\subsection{Effect of seed treatment with inducers of Penicillium sp. RFUOM 14 on seed germination and seedling vigor}

Seeds treated with inducers of phosphate solubilizing fungus Penicillium sp. RFUOM 14 were analyzed for their effect on seed germination and seedling vigor of selected crop plants. The treatment of conidial suspension and culture filtrate of Penicillium sp. RFUOM 14 to the seeds of different crop plants enhanced the seed germination and seedling vigor when compared to respective control sets.

Among the different time intervals of treatment, inducers treated for $6 \mathrm{~h}$ offered maximum enhancement in seed germination and seedling vigor when compared to $3 \mathrm{~h}$ treated seedlings.

A maximum of $86 \%, 80 \%$ and $83 \%$ of seed germination and seedling vigor $985.25,523$ and 673.5 was observed in pearl millet, brinjal and tomato seedlings, respectively when treated as conidial suspension for $6 \mathrm{~h}$ (Table $2 \& 3$ ). The culture filtrate treatment of Penicillium sp. RFUOM 14 also enhanced seed germination and seedling vigor but was lower when compared to conidial suspension treatment for $6 \mathrm{~h}$ (Fig. 2).
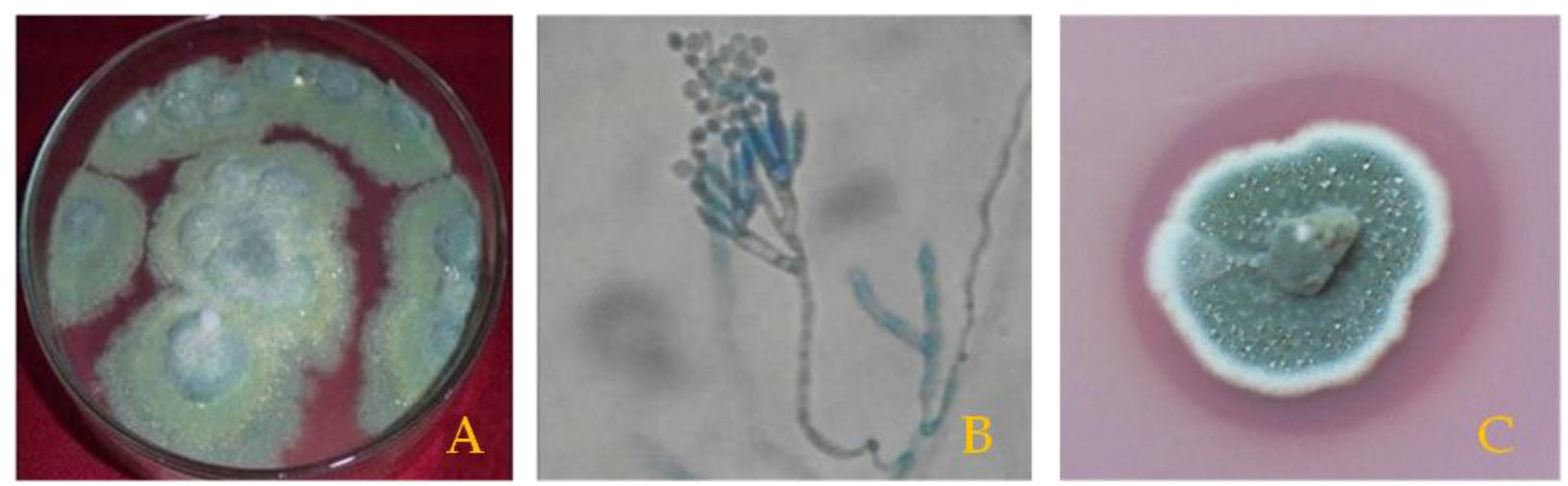

Fig 1: Phosphate solubilizing Penicillium sp. RFUOM 14. A- Colony morphology; B- Conidia under Microscope; C- Zone of phosphate solubilization on Pikovskaya plates.
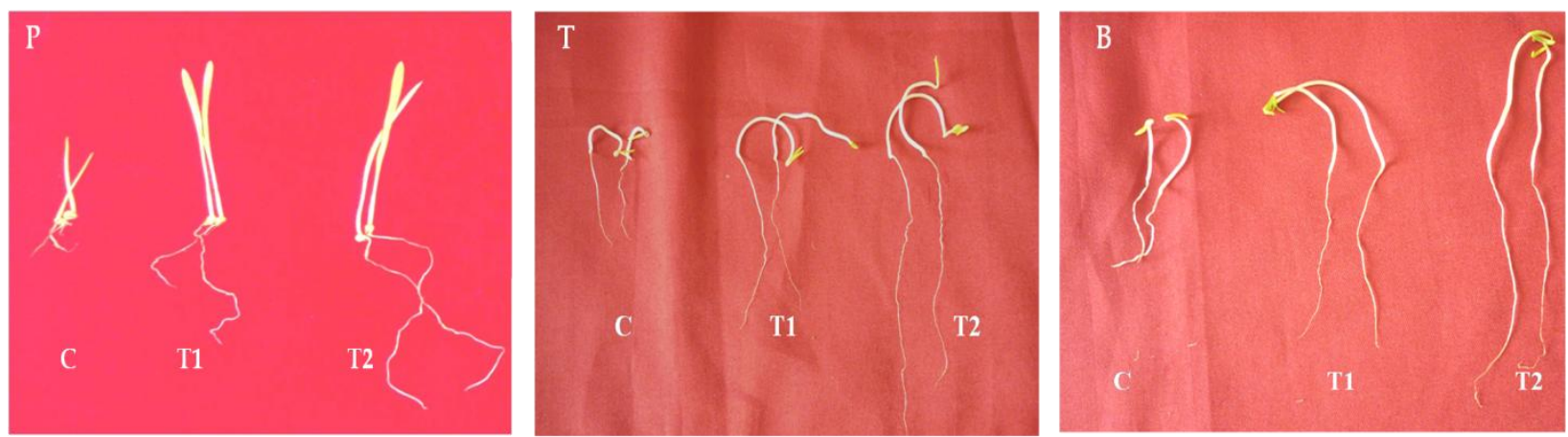

Fig 2: Seed treatment with Penicillium sp. RFUOM 14 on seed germination and vigor. P-Pearl millet; T- Tomato; B- Brinjal; C- Control; T1- CF; T2- CS. 
Table 2: Effect of seed treatment with inducers Penicillium sp. RFUOM 14 on seed germination and vigor after $3 \mathrm{~h}$.

\begin{tabular}{|c|c|c|c|c|c|c|c|}
\hline \multirow{2}{*}{ Fungi } & \multirow{2}{*}{ Treatment } & \multicolumn{2}{|c|}{ Pearl millet } & \multicolumn{2}{|c|}{ Brinjal } & \multicolumn{2}{|c|}{ Tomato } \\
\hline & & SG & SV & SG & SV & SG & SV \\
\hline Penicillium sp. & Conidial suspension & $81.25 \pm 0.47^{\mathrm{a}}$ & $847.25 \pm 5.43^{\mathrm{a}}$ & $75.00 \pm 0.81^{\mathrm{a}}$ & $488.50 \pm 6.02^{\mathrm{a}}$ & $81.00 \pm 0.70^{\mathrm{a}}$ & $670.00 \pm 4.84^{\mathrm{a}}$ \\
\hline RF UOM 14 & Culture Filtrate & $78.25 \pm 0.62^{b}$ & $803.50 \pm 4.78^{b}$ & $72.00 \pm 0.81^{\mathrm{b}}$ & $460.50 \pm 4.19^{b}$ & $79.50 \pm 1.04^{\mathrm{b}}$ & $641.50 \pm 4.19^{b}$ \\
\hline Control & & $72.75 \pm 0.85^{\mathrm{c}}$ & $763.25 \pm 6.01^{c}$ & $70.00 \pm 0.40^{\mathrm{b}}$ & $440.50 \pm 3.86^{\mathrm{c}}$ & $77.00 \pm 0.81^{\mathrm{c}}$ & $620.50 \pm 4.64^{c}$ \\
\hline
\end{tabular}

Values are means of four independent replicates. Means followed by the same letter(s) within the same column are not significantly ( $p \leq 0.05)$ different according to Tukey's HSD.

Table 3: Effect of seed treatment with inducers of Penicillium sp. RFUOM 14 on seed germination and vigor after $6 \mathrm{~h}$.

\begin{tabular}{|c|c|c|c|c|c|c|c|}
\hline \multirow{2}{*}{ Fungi } & \multirow{2}{*}{ Treatment } & \multicolumn{2}{|c|}{ Pearl millet } & \multicolumn{2}{|c|}{ Brinjal } & \multicolumn{2}{|c|}{ Tomato } \\
\hline & & SG & SV & SG & SV & SG & SV \\
\hline Penicillium sp. & Conidial suspension & $85.75 \pm 0.62^{\mathrm{a}}$ & $985.25 \pm 4.49^{\mathrm{a}}$ & $80.00 \pm 0.70^{\mathrm{a}}$ & $523.00 \pm 2.48^{\mathrm{a}}$ & $83.00 \pm 0.81^{\mathrm{a}}$ & $673.50 \pm 3.17^{\mathrm{a}}$ \\
\hline RF UOM 14 & Culture Filtrate & $80.00 \pm 0.81^{\mathrm{b}}$ & $962.00 \pm 4.32^{\mathrm{b}}$ & $74.00 \pm 0.81^{\mathrm{b}}$ & $492.00 \pm 4.32^{\mathrm{b}}$ & $80.00 \pm 0.70^{\mathrm{b}}$ & $662.00 \pm 3.36^{\mathrm{a}}$ \\
\hline Control & & $72.75 \pm 0.85^{\mathrm{c}}$ & $763.25 \pm 6.01^{\mathrm{c}}$ & $70.00 \pm 0.40^{\mathrm{c}}$ & $440.50 \pm 3.86^{\mathrm{c}}$ & $77.00 \pm 0.81^{\mathrm{c}}$ & $620.50 \pm 4.64^{b}$ \\
\hline
\end{tabular}

Values are means of four independent replicates. Means followed by the same letter(s) within the same column are not significantly ( $p \leq 0.05)$ different according to Tukey's HSD.

\section{DISCUSSION}

Phosphorus is the second important key element after nitrogen as a mineral nutrient in terms of quantitative plant requirement. Although phosphates are abundant in soils, in both organic and inorganic forms, its availability is restricted as it occurs mostly in insoluble forms. To satisfy crop nutritional requirements, $\mathrm{P}$ is usually added to soil as chemical $\mathrm{P}$ fertilizer, however synthesis of chemical $\mathrm{P}$ fertilizer is highly energy intensive processes and has long term impacts on the environment in terms of eutrophication, soil fertility depletion, carbon footprint. Moreover, plants can use only a small amount of this P since 75$90 \%$ of added $\mathrm{P}$ is precipitated by metal cation complexes, and rapidly becomes fixed in soils [16]. Such environmental concerns have led to the search for sustainable way of $\mathrm{P}$ nutrition of crops. In this regards phosphate-solubilizing microorganisms (PSM) have been seen as best eco-friendly means for $\mathrm{P}$ nutrition of crop. Although, several bacterial (Pseudomonads and Bacilli) and fungal strains (Aspergillus sp. and Penicillium sp.) have been identified as PSMs [16, 17] and their performance under in situ conditions is not reliable and therefore needs to be improved by using either genetically modified strains or co-inoculation techniques.

In the present investigation, fungi were isolated from rhizosphere soil of various crop plants. A total of 22 fungi were isolated from the rhizosphere soil and screened for their ability to solubilize phosphates in PKV medium. Of these 22 rhizosphere fungi, only Penicillium sp. RF UOM 14 was found positive for phosphate solubilization. Similarly, there are reports on both bacterial and fungal strains exhibiting $\mathrm{P}$ solubilizing activity by the formation of clear halo zone (a sign of solubilization) around their colonies [16]. The results correlate with the findings of Mendes et al. [18], where isolates of Aspergillus niger FS1, Penicillium canescens FS23 and Eupenicillium ludwigii were able to solubilize all forms of $\mathrm{P}$. The phosphate-solubilizing microbes showing greater solubilization (both qualitatively and quantitatively) of insoluble $\mathrm{P}$ under in vitro conditions were selected for field trials prior to production in bulk for ultimate transmission as a biofertilizer [16]. It is also suggested that phosphate solubilization, production of IAA, and other related compounds by the fungus will interact with plants as part of its colonization, leading to growth promotion, induced resistance, and modification of basal plant defense mechanisms [19-22].

Further, in the present study, conidial suspension and culture filtrate of the phosphate solubilizing fungi Penicillium sp. RFUOM 14 was used as seed treatment to different crop plants. In comparison with the SDW control, a significant enhancement of seed germination and vigour was noticed in phosphate solubilizing fungi Penicillium sp. RFUOM 14, with maximum germination of $86 \%$ and seedling vigour index score of 985 in pearl millet. Similarly, there was also significant increase in seed germination and vigor in tomato and brinjal plants also. Sudisha et al. [23] reported that, tomato seeds treated with phosphate solubilizing fungi like Trichoderma, Penicillium, etc., enhanced seed germination and seedling vigor. Similarly, A. niger and $P$. italicum solubilize tri-calcium phosphate (TCP) and improve the growth of soyabean in TCP amended soil [24]. Likewise, seeds treated with T. harzianum in sunflower [25] and Penicillium chrysogenum and $P$. oxalicum in pearl millet $[13,26]$ which were isolated from the rhizosphere soil offered enhanced seed germination and seedling vigour over the control.

\section{CONCLUSIONS}

Nowadays, PSM technology largely depends on the development and distribution of good quality inoculants to farming communities. Therefore, there is a need for extensive and consistent research efforts to identify and characterize more PSM with greater efficiency for their ultimate application under field conditions. Hence the results of the present study may contribute to the findings of other researchers, where we were able to notice enhancement in seed germination and vigor of crop plants when treated with conidial suspension and culture filtrate of phosphate solubilizing fungi Penicillium sp. RFUOM 14. Furthermore, 
researchers need to address certain issues, like how to improve the efficacy of biofertilizers, what should be an ideal and universal delivery system, how to stabilize these microbes in soil systems, and how nutritional and root exudation aspects could be controlled in order to get maximum benefits from PSM application.

\section{ACKNOWLEDGEMENTS}

Financial support and sponsorship: The first author is thankful to UGC, Govt. of India, New Delhi, for awarding Post-Doctoral Fellowship. MCT is thankful to CSIR, Govt. of India, New Delhi for Research Associate Fellowship. The Corresponding author (KNA) is grateful to DBT Project on the development of bioformulation from PGPF and PGPR.

Conflict of Interests: There are no conflicts of interest.

\section{REFERENCES}

1. Reena T, Dhanya H, Deepthi MS, Pravitha D. Isolation of Phosphate Solubilizing bacteria and fungi from rhizospheres soil from banana plants and its effect on the growth of Amaranthus cruentus L. IOSR Journal of Pharmacy and Biological. 2013; 5:06-11.

2. Sharma S, Vijay Kumar, Tripathi RB. Isolation of Phosphate Solubilizing Microorganism (PSMs) from soil. J. Microbiololgy Biotechnology Research. 2011; 1(2):90-95.

3. Richardson AE. Making microorganisms mobilize soil phosphorus. In: E. Velázquez and C. Rodríguez-Barrueco (eds.) First International Meeting on Microbial Phosphate Solubilization. 2007; pp. 85-90

4. Pradhan N, Sukla LB. Solubilization of inorganic phosphates by fungi isolated from agriculture soil. African Journal of Biotechnology. 2005; 5:850-854.

5. Sundara B, Natarajan V, Hari K. Influence of phosphorus solubilizing bacteria on the changes in soil available phosphorus and sugar cane and sugar yields. Field Crops Research. 2002; 77:43-49.

6. Nahas E. Factors determining rock phosphate solubilization by microorganisms isolated from soil. World J. Microbiolology Biotechnology. 1996; 12:567-572.

7. Whitelaw MA. Growth promotion of plants inoculated with phosphate solubilizing fungi. 1999; 69:99-151.

8. Han HS, Supanjani, Lee KD. Effect of co-inoculation with phosphate and potassium solubilizing bacteria on mineral uptake and growth of pepper and cucumber. Plant Soil Environment. 2006; 52:130-136.

9. Harman GE. Multifunctional fungal plant symbionts: new tools to enhance plant growth and productivity. New Phytologist. 2011; 189(3):647-649.

10. Murali M, Amruthesh KN, Sudisha J, Niranjana SR, Shetty HS. Screening for plant growth promoting fungi and their ability for growth promotion and induction of resistance in pearl millet against downy mildew disease. 2012; 4(5):30-36.

11. Pikovskaya RI. Mobilization of phosphorus in soil in connection with vital activity of some microbial species. Mikrobiologiya. 1948; 17(362):e370.

12. Niranjana SR, Lalitha S, Hariprasad P. Mass multiplication and formulations of biocontrol agents against Fusarium wilt of pigeonpea through seed treatment. International Journal of Pest Management. 2009; 55(4):317-324.

13. Murali M, Amruthesh KN. Plant Growth-promoting Fungus Penicillium oxalicum enhances plant growth and induces resistance in pearl millet against downy mildew disease. Journal of Phytopathology. 2015; 163(9):743-754.
14. Singh SD, Gopinath R. A seedling inoculation technique for detecting downy mildew resistance in pearl millet. Plant Disease. $1985 ; 72: 425-428$

15. Abdul Baki, Anderson JD. Vigor analysis in Soybean seed by multiple criteria. Crop Science. 1973; 13:630-633.

16. Sharma SB, Sayyed RZ, Trivedi MH, Gobi, TA. Phosphate solubilizing microbes: sustainable approach for managing phosphorus deficiency in agricultural soils. SpringerPlus. 2013; 2(1):1.

17. Khan MR, Khan SM. Effect of root-dip treatment with certain phosphate solubilizing microorganisms. Bioresour Technology. 2002; 85(2):213-215.

18. Mendes GO, Dias CS, Silva IR, Junior JIR, Pereira OL, Costa MD. Fungal rock phosphate solubilization using sugarcane bagasse. World J Microbiology Biotechnology. 2013; 29:43-50.

19. Altomare C, Norvell WA, Bjorkman T, Harman GE. Solubilization of phosphates and micronutrients by the plant growth promoting and biocontrol fungus Trichoderma harzianum Rifai 1295-22. Applied and Environmental Microbiology. 1999; 65:2926-2933.

20. Harman GE, Howell CR, Viterbo A, Chet I, Lorito M. Trichoderma species- opportunistic, avirulent plant symbionts. Nature Reviews Microbiology. 2004; 2:43-56

21. Contreras-Cornejo H, Macias-Rodriguez L, Cortes-Penago, LopezBuci, J. Trichoderma virens, a plant beneficial fungus, enhances biomass production and promotes lateral root growth through an auxin-dependent mechanism in Arabidopsis. Plant Physiology. 2009; 149:1579-1592.

22. Yadav J, Verma JP, Tiwari KN. Plant growth promoting activities of fungi and their effect on chickpea plant growth. Asian Journal of Biological Sciences. 2011; 4:291-299.

23. Sudisha J, Mostafa M, Lam-Son PT, Ito SI. Characterization of rhizosphere fungi that mediate resistance in tomato against bacterial wilt disease. Journal of Experimental Botany. 2013; 64(12):3829. 3842 .

24. Iman M, Azouni EI. Effect of phosphate solubilizing fungi on growth and nutrient uptake of soyabean plants. J. Applied Scientific Research. 2008; 4(6):592-598.

25. Nagaraju A, Murali M, Sudisha J, Amruthesh KN, Murthy SM. Beneficial microbes promote plant growth and induce systemic resistance in sunflower against downy mildew disease caused by Plasmopara halstedii. Current Botany. 2013; 3(5):12-18

26. Murali M, Sudisha J, Amruthesh KN, Ito SI, Shetty HS. Rhizosphere fungus Penicillium chrysogenum promotes growth and induces defence-related genes and downy mildew disease resistance in pearl millet. Plant Biology. 2013; 15(1):111-118.

\section{How to cite this article:}

Murali M, Thriveni MC, Manjula S, Mythrashree SR, Amruthesh KN. Isolation of phosphate solubilizing fungi from rhizosphere soil and its effect on seed growth parameters of different crop plants. J App Biol Biotech. 2016; 4 (06): 022026. DOI: $10.7324 / \mathrm{JABB} .2016 .40604$ 